\title{
ANÁLISE DOS ASPETOS AMBIENTAIS EM UNIDADES DE ALIMENTAÇÃO COLETIVA DOS SERVIÇOS DE AÇÃO SOCIAL DA UNIVERSIDADE DO PORTO
}

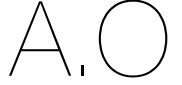

ARTIGO ORIGINAL

1 Faculdade de Ciências da Nutrição e Alimentação da Universidade do Porto, Rua Dr. Roberto Frias, 4200-465 Porto, Portugal

2 Universidade Federal de Santa Catarina,

Rua Eng. Agronômico Andrei Cristian Ferreira, $\mathrm{s} / \mathrm{n}$ - Trindade, Florianópolis, 88040-900, Brasil

3 GreenUPorto, Campus de Vairão, Edifício de Ciências Agrárias,

Rua da Agrária, $\mathrm{n} \circ 747$ 4485-646 Vairão, Portuga

4 LAQV@REQUIMTE, Rua Dr. Roberto Frias, 4200-465 Porto, Portugal

"Endereço para correspondência:

da Roch

Faculdade de Ciências da Nutriçāo e Alimentaçāo da Universidade do Porto Rua Dr. Roberto Frias, 4200-465 Porto, Portuga adarocha@fcna.up.pt

Histórico do artigo:

Recebido a 24 de fevereiro de 2019 Aceite em 26 de outubro de 2019

\section{ANALYSIS OF THE ENVIRONMENTAL ASPECTS IN FOOD UNITS OF UNIVERSITY OF PORTO SOCIAL SERVICES}

RESUMO

Ao longo das últimas décadas, devido às alterações do estilo de vida da população, a alimentação tem-se tornado cada vez menos sustentável.

O presente estudo teve como objetivo identificar as melhorias relacionadas com a sustentabilidade, com ênfase nos aspetos ambientais, das 11 unidades de alimentação dos Serviços de Alimentação dos Serviços Sociais de uma Universidade Portuguesa. Para avaliar os aspetos ambientais destas unidades foi criada uma checklist, contendo 72 questões, que permitem avaliar qualitativamente as unidades acerca do seu desempenho ambiental, a nível de: consumos de água, energia elétrica e gás; gestão de produtos químicos e resíduos; utilização de hortofrutícolas e ainda a nível da satisfação do cliente.

A recolha foi realizada através de visitas às cozinhas e restantes espaços das unidades de alimentação, através da observação direta pela pesquisadora e respetivo registo.

Todas as questões foram pontuadas através de uma adaptação do método de Failure Mode and Effect Analysis (FMEA). Foram classificadas numa escala de 1 a 3, sendo o valor "3" atribuído a aspetos adequados, ou seja, é a opção com menor impacto ambiental face a determinada situação e o valor "1" representa a situação que terá maior impacto ambiental, obtendo-se uma avaliação qualitativa dos aspetos ambientais de cada uma das unidades.

As classificações obtidas nas diversas unidades encontram-se no intervalo de 55\% a 74\%, que corresponde à classificação de Aceitável. Os responsáveis das unidades não conhecem os valores gastos em água, eletricidade e gás não tendo estabelecidas metas de gastos de forma a reduzir o desperdício ou controlar os gastos. Na maioria das unidades é feita a manutenção preventiva dos equipamentos. A maioria das unidades localiza-se em edifícios antigos não possuindo classificação energética. Todos os produtos de higienização eram maioritariamente não-biodegradáveis mas possuem ficha técnica e dados de segurança. A maioria das unidades faz a separação de resíduos e a reciclagem de óleos, paletes e outros. Em nenhuma das unidades é registada a quantidade de sobras. Nenhuma das unidades possui trituradora de resíduos orgânicos ou inorgânicos e todas utilizam produtos/ materiais descartáveis, Através da análise dos resultados obtidos na checklist, concluiu-se que todas se encontravam acima do limite de Aceitável. No entanto de uma forma geral são necessários investimentos económicos, bem como investimentos a nível de mudanças de mentalidade e atitudes de trabalhadores e gestores, tendo em vista a melhoria dos aspetos ambientais destas unidades.

\section{PALAVRAS-CHAVE}

Ambiente, Avaliação, Consumíveis, Resíduos

ABSTRACT

In the last few decades, due to several changes in population's life style, food has become more and more unsustainable. The main objective of the present study was to identify the needs in terms of sustainability, mainly focusing environmental aspects, of the 11 canteens of the Food Service Units of a Portuguese University

To assess the environmental aspects of canteens, a checklist was created, containing 72 questions which allowed the qualitative evaluation of the environmental performance of food units, in terms of water, electricity and gas consumption; chemical and waste management; use of horticultural products and customer satisfaction.

Data collection was performed during visits to the canteens through direct observation by the researcher and recording of the situations contained in the checklist.

Each question was scored, using an adaptation of Failure Mode and Effect Analysis (FMEA) method. Questions were score on a scale of 1 to 3, were " 3 " was assigned to suitable aspects, corresponding to the option with less environmental impact and " 1 " to the highest environmental impact, obtaining a qualitative assessment of the environmental aspects of each canteen evaluated. The ratings obtained by the different canteens were in the range of 55\% to $74 \%$, corresponding to the classification of Acceptable. The managers are not aware of the amounts spent on water, electricity and gas, neither targets in order to reduce or waste or control expenses. In most units, preventive maintenance of equipment is carried out. Most units are located in old buildings and do not have an energy rating. All hygiene products were mostly non-biodegradable, but had technical sheets including safety data. Most units select waste and recycle oils, pallets and others. None of the units has a quantity of leftovers registered. None of the units has an organic or inorganic waste shredder and all use disposable materials. 
Through the analyses of the checklist' results, it was possible to conclude that all canteens were above the Acceptable limit. Nevertheless, the general state points to the need of economic investments as well as attitudes and behavior changes of workers and managers in order to increase sustainability in terms of environmental aspects of the canteens.

KEYWORDS

Environment, Evaluation, Consumables, Waste

\section{INTRODUÇÃO}

A produção de refeições é uma das mais importantes atividades em alimentação coletiva. Envolve uma série de processos desde a seleção e armazenamento de alimentos à produção da refeição, decorrendo quase como um processo industrial $(1,2)$.

Paralelamente há ainda a considerar as atividades de limpeza e higienização das instalações, equipamentos e utensílios. Em todas estas etapas existem processos com impacto ambiental, onde se inclui a geração de resíduos procedentes dos alimentos, bem como das embalagens descartáveis utilizadas, eliminação inadequada de produtos e embalagens, utilização de produtos não-biodegradáveis e desperdício de recursos, incluindo o consumo excessivo de água e energia (2-5).

Num serviço desta natureza, antes da refeição ser consumida, pode já ter ocorrido desperdício de recursos naturais e especialmente de alimentos com consequentemente geração de resíduos (2).

Identificar e compreender os aspetos ambientais relacionados com a produção de refeições é o primeiro passo para a melhoria da sustentabilidade em alimentação coletiva.

Nos serviços de alimentação coletiva, os desperdícios orgânicos gerados podem ocorrer quer no processo produtivo quer na distribuição das refeições, estando ainda associado ao desperdício dos recursos, que foram necessários para produzir alimentos que não foram consumidos, como água, energia e solo fértil (5).

A alimentação tem um forte impacto nestes recursos, sendo mais diretamente, tanto a água como a energia elétrica, recursos indispensáveis ao processo de produção de refeições e seu fornecimento $(2,6)$.

A American Dietetic Association e a Academy of Nutrition and Dietetics consideram, uma vez que os Nutricionistas trabalham em ambientes onde os seus esforços a nível da gestão podem minimizar desperdícios, tanto alimentares como de recursos, e suportar a sustentabilidade do sistema alimentar, devem estar conscientes das implicações globais das suas ações e têm a responsabilidade de garantir que estas sejam o mais sustentável possível. Como tal foram estabelecidos requisitos orientadores em relação à gestão $(7,8)$.

A água é utilizada dentro das unidades em todas as operações, podendo o custo hídrico de uma refeição oscilar entre os $11 \mathrm{~L}$ e os 602,7L. Com este tipo de variação, diretamente associado às múltiplas variáveis existentes numa unidade, tentar reduzir o seu gasto é de extrema importância, não só nas operações como com a própria ementa (por exemplo reduzir a oferta de carnes vermelhas e laticínios, aumentar o de hortofrutícolas, principalmente de produção local, entre outros) (9-11).

De acordo com a American Dietetic Association para a redução dos gastos de água é recomendado o reaproveitamento da água de cozimento, utilização de produtos de limpeza biodegradáveis e a minimização de resíduos descartados na rede sanitária (7).

A alimentação humana é responsável por 30\% do consumo energético a nível mundial (12). Os gastos elétricos por refeição podem variar entre os 0,2kw/h e os $1,3 \mathrm{kw} / \mathrm{h}$ de consumo faturado o que corresponde a valores de emissão de $\mathrm{CO}_{2}$ entre os $20,26 \mathrm{~g} \mathrm{CO}_{2}$ a $131,69 \mathrm{~g} \mathrm{CO}(11,13,14)$.
De acordo com a American Dietetic Association para a redução dos gastos de energia elétrica é recomendado a escolha de equipamentos mais eficientes, a criação de estratégias de poupança energética e a realização de manutenção preventiva dos equipamentos (7).

A higienização das instalações, equipamentos, superfícies e utensílios é parte integrante da garantia da segurança alimentar. Na década de 50 surgiram os detergentes biodegradáveis criados para se degradarem na água após utilização, mas com estes surgiu o problema dos fosfatos livres, que causaram eutrofização excessiva. Atualmente os detergentes considerados verdadeiramente biodegradáveis substituíram estes compostos por surfactantes iónicos (15-17).

A redução na produção de resíduos e no consumo de recursos são das primeiras táticas de gestão ambiental a serem implementadas uma vez que reduzem os gastos e não implicam investimentos significativos além de trazerem benefícios fiscais imediatos. Estratégias de gestão tanto da qualidade como ambiental podem ser benéficas para a empresa a nível económico e da gestão de tempo $(18,19)$.

A avaliação da gestão ambiental está intimamente relacionada com a gestão da qualidade e visa proporcionar um processo de mudança organizacional e de melhoria contínua da qualidade ambiental. A maioria das empresas utiliza os pontos avaliados pelas Green Restaurant Association que contempla a avaliação de sete categorias: 1. "Eficiência no uso de água"; 2 . "Redução de resíduos"; 3 . "Mobiliário sustentável e materiais de construção"; 4. "Comida sustentável"; 5. "Energia (equipamentos com selo de eficiência e uso de fontes de energia limpa)"; 6. "Utilização de descartáveis"; 7. "Produtos químicos e redução de poluição" (20-22).

Controlar estes problemas torna-se essencial em alimentação coletiva, pois a elevada quantidade de refeições servidas diariamente, determinam que um mau planeamento do sistema tenha repercussões diretas sobre as questões ambientais, no entanto não existem propriamente especificações sobre "de que forma" isto deve ser feito ou qual será a forma mais correta de o fazer (3).

A utilização de uma checklist para recolha e análise de informação a nível dos espaços de produção de refeições pode auxiliar a identificar o estado das práticas ambientais da unidade e definir as prioridades de intervenção tendo em vista a sua otimização. Pode assim constituir uma importante ferramenta para a gestão, uma vez que reduzindo os consumos, também os gastos fixos da unidade irão ser reduzidos, fazendo com que os aspetos ambientais significativos sejam uma verdadeira prioridade para o gestor da unidade, levando a uma redução da depleção de recursos e melhoria geral do ecossistema onde se inserem (23).

As unidades de alimentação coletiva como grandes fornecedores de refeições, tem um forte impacto no ambiente devido à produção de resíduos sólidos urbanos, poluíção atmosférica, consumo de energia e consumo de água. Uma vez que a responsabilidade social é o elo de ligação entre estas e a população, estas têm a responsabilidade de atingir um desenvolvimento sustentável $(24,25)$.

O objetivo deste trabalho foi analisar os aspetos ambientais em unidades de alimentação coletiva dos Serviços de Alimentação dos Serviços Sociais da Universidade do Porto (SASUP) e identificar prioridades de intervenção para diminuir o impacto ambiental das unidades.

\section{METODOLOGIA}

Trata-se de uma pesquisa de campo, aplicada transversal e descritiva, com análise qualitativa das variáveis. O objeto de estudo são os aspetos ambientais das 11 unidades de alimentação dos Serviços de Ação Social da Universidade do Porto (SASUP), cuja missão é 
prestar serviços no âmbito da alimentação, nutrição e segurança alimentar, assegurando o equilíbrio nutricional e a qualidade alimentar das refeições $(26,27)$.

Os SASUP têm 88 funcionários e serviu em 2015546.949 refeições, distribuídas pelas 11 unidades, onde apenas 2 servem jantares. $O$ custo de uma refeição social nas cantinas da Universidade do Porto é de $2,65 €$, sendo que em apenas uma das unidades este valor não é inferior ao custo de produção. As unidades de alimentação são a opção mais económica, dentro da comunidade académica, para os estudantes consumirem as suas refeições diárias (27).

Todas as informações relativamente às unidades que não foi possível obter in loco foram obtidas através da plataforma dos Serviços de Alimentação dos SASUP. Sendo que não foi possível obter informação mais recente. $\mathrm{O}$ acesso às cantinas, registo e utilização de informações obtidas foram autorizados pelos SASUP.

Foi criada uma checklist para avaliar qualitativamente os aspetos ambientais destas unidades, com base em indicadores ambientais definidos após revisão bibliográfica (28-33) distribuídos por 7 itens: 1) Consumo de Água (17 questões); 2) Consumo de Eletricidade (15 questões); 3) Consumo de Gás (11 questões); 4) Gestão de Produtos Químicos (7 questões); 5) Gestão de Resíduos (13 questões); 6) Utilização de Hortofrutícolas (3 questões); 7) Avaliação da Satisfação do Utente (6 questões), num total de 72 questões.

O sistema de pontuação utilizado foi uma adaptação do método de Failure Mode and Effect Analysis (FMEA) utilizado por Zambrano e Martins (34) para classificar a abrangência dos impactos ambientais. As ocorrências foram classificadas numa escala de 1 a 3, o valor "3" é atribuído a aspetos adequados, ou seja, é a opção que terá menor impacto ambiental, face a determinada situação analisada. O valor "2" representa um impacto ambiental intermédio, tendo sido excluído de questões de "sim ou não". O valor "1" representa a situação com maior impacto ambiental, sendo a menos adequada para a situação em causa. Na Tabela 1, é apresentado um exemplo desta distribuição. A aplicação da checklist ocorreu durante as visitas efetuadas às 11 unidades de alimentação dos SASUP, sendo que cada unidade apenas foi visitada uma vez, tendo em conta os itens a avaliar pela checklist. A aplicação foi realizada através da observação direta pela pesquisadora e o registo das situações contidas na checklist. No entanto questões sobre a manutenção preventiva ou existência de registos de consumos, foram feitas diretamente aos responsáveis das cozinhas, os funcionários e o pessoal responsável pela manutenção dos espaços e equipamentos.

As unidades de alimentação foram codificadas de forma a manter o sigilo dos resultados.

Cada questão foi pontuada, obtendo-se uma avaliação qualitativa do estado ambiental de cada uma das unidades de alimentação avaliadas e classificadas mediante a seguinte grelha de classificação (Tabela 2). A avaliação qualitativa foi obtida comparando os resultados obtidos por cada unidade, frente à aplicação da mesma checklist em situação de excelência, ou seja, realizou-se o somatório das classificações da checklist aplicada e comparou-se com o valor que essa checklist obteria caso todos os itens obtivessem classificação máxima, sendo esse o nosso valor de padrão (Tabela 3).

De salvaguardar que os valores apresentados sobre cada unidade são valores obtidos através do Relatório de Contas de 2015 (27), uma vez que não foi possível obter dados mais recentes nem mais completos, pois não foi possível ter acesso aos valores de consumos faturados, assim sendo, não podemos aferir quando foi gasto o quê nem como. Além disso, cinco das onze cantinas não possuem contador próprio, sendo que o partilham com snack-bares e/ou grill. Nestes casos, mesmo
Tabela 1

Exemplo de pergunta presente na checklist e distribuição da pontuação adotada

\begin{tabular}{|c|c|}
\hline Automática & (3 pontos) \\
\hline Acionada com pedal & (2 pontos) \\
\hline Manual & (1 ponto) \\
\hline Não aplicável & - \\
\hline
\end{tabular}

\section{Tabela 2}

Classificação da avaliação qualitativa aplicada à checklist

\begin{tabular}{lc}
\multicolumn{1}{c}{ CLASSIFICAÇÃO } & VALOR PERCENTUAL (\%) \\
\hline Muito Bom & $90-100$ \\
\hline Bom & $75-89$ \\
\hline Aceitável & $50-74$ \\
\hline Não Aceitável & $\leq 49$ \\
\hline
\end{tabular}

\section{Tabela 3}

Valores usados para o cálculo da classificação da checklist

\begin{tabular}{ccccc}
$\begin{array}{c}\text { UNIDADE DE } \\
\text { ALIMENTAÇÃO }\end{array}$ & $\begin{array}{c}\text { PONTUAÇÃO } \\
\text { OBTIDA } \\
\text { EM VALOR } \\
\text { ABSOLUTO }\end{array}$ & $\begin{array}{c}\text { PONTUAÇÃO } \\
\text { OBTIDO EM \% }\end{array}$ & $\begin{array}{c}\text { PONTUAÇÃO } \\
\text { MÁXIMA } \\
\text { EM VALOR } \\
\text { ABSOLUTO }\end{array}$ & $\begin{array}{c}\text { PONTUAÇÃO } \\
\text { MÁXIMA EM \% }\end{array}$ \\
\hline A & 347 & 68 & 513 & 100 \\
\hline B & 406 & 67 & 606 & 100 \\
\hline C & 516 & 63 & 822 & 100 \\
\hline D & 381 & 70 & 546 & 100 \\
\hline E & 321 & 66 & 486 & 100 \\
\hline F & 166 & 62 & 267 & 100 \\
\hline G & 237 & 74 & 321 & 100 \\
\hline H & 446 & 55 & 810 & 100 \\
\hline I & 115 & 70 & 222 & 100 \\
\hline J & 255 & 73 & 348 & 100 \\
\hline L & 367 & 70 & 522 & 100 \\
\hline & & & & \\
\hline
\end{tabular}

tendo acesso aos valores de consumo, não poderíamos aferir se o gasto na produção de refeições por parte da cantina foi elevado ou não.

\section{RESULTADOS E DISCUSSÃO DOS RESULTADOS}

As 11 unidades de alimentação onde foi aplicada a checklist apresentam condições estruturais distintas, tanto a nível de espaço físico como de equipamentos. Apesar dos procedimentos serem praticamente transversais estas apresentam resultados a nível dos aspetos ambientais muito díspares. A classificação obtida pelas diversas unidades encontra-se no intervalo de 55\% a 74\% (Aceitável) (Tabela 4).

Com base nestes valores apenas podemos aferir que em todos as unidades há melhorias a serem implementadas. Assim sendo analisámos as checklist de duas formas; numa primeira abordagem analisámos os valores e as respostas dadas pelas unidades às múltiplas questões da checklist e particularizaram-se as exceções, tanto positivas como negativas e por fim analisamos as oportunidades de melhoria.

Nenhuma das unidades de alimentação mantem o registo dos gastos de água, de eletricidade e gás. Os únicos registos são faturas para efeitos fiscais.

Algumas unidades de alimentação partilham os contadores de água e de eletricidade com a faculdade onde estão inseridas (unidades $F$, $\mathrm{G}, \mathrm{H}, \mathrm{I}$ e J).

Os responsáveis das unidades não conhecem os valores gastos em água, eletricidade e gás não tendo estabelecidas metas de gastos de forma a reduzir o desperdício ou controlar os gastos. A criação 


\begin{tabular}{|c|c|c|c|c|c|c|c|c|}
\hline \multirow[b]{2}{*}{$\begin{array}{l}\text { UNIDADE DE } \\
\text { ALIMENTAÇĀO }\end{array}$} & \multicolumn{8}{|c|}{ PONTUAÇÃO EM \% } \\
\hline & GERAL & $\begin{array}{l}\text { CONSUMO } \\
\text { DE ÁGUA }\end{array}$ & $\begin{array}{l}\text { CONSUMO DE } \\
\text { ELETRICIDADE }\end{array}$ & CONSUMO DE GÁS & $\begin{array}{l}\text { GESTÃO DE } \\
\text { PRODUTOS } \\
\text { QUÍMICOS }\end{array}$ & $\begin{array}{l}\text { GESTÃO DE } \\
\text { RESÍDUOS }\end{array}$ & $\begin{array}{l}\text { UTILIZAÇÃO DE } \\
\text { HORTOFRUTÍCOLAS }\end{array}$ & $\begin{array}{l}\text { AVALIAÇÃO DA } \\
\text { SATISFAÇÃO DO } \\
\text { UTENTE }\end{array}$ \\
\hline A & 68 & 65 & 73 & 56 & 78 & 60 & 68 & 83 \\
\hline B & 67 & 71 & 70 & 54 & 72 & 60 & 67 & 87 \\
\hline C & 63 & 65 & 61 & 61 & 72 & 60 & 67 & 67 \\
\hline D & 70 & 66 & 84 & 54 & 72 & 60 & 67 & 87 \\
\hline E & 66 & 60 & 71 & 55 & 72 & 60 & 68 & 67 \\
\hline $\mathrm{F}$ & 62 & 62 & 53 & 50 & 63 & 60 & 68 & 100 \\
\hline G & 74 & 67 & 76 & N/A & 71 & 50 & 83 & 100 \\
\hline $\mathrm{H}$ & 55 & 56 & 50 & 51 & 74 & 60 & 68 & 83 \\
\hline 1 & 70 & 67 & 62 & N/A & 71 & 50 & 83 & 87 \\
\hline$J$ & 73 & 71 & 76 & 59 & 78 & 40 & 83 & 100 \\
\hline L & 70 & 67 & 80 & 61 & 71 & 60 & 68 & 83 \\
\hline
\end{tabular}

A sigla N/A corresponde a "Não Aplicável"

de metas para os gastos e a consciencialização dos responsáveis das unidades segundo Mikkola (35), será "a forma mais fácil de implementar mudanças nas unidades é através da disposição dos colaboradores de serem sustentáveis e por meio da criação de metas a serem cumpridas podemos "cultivar" a vontade de melhoria".

Na maioria das unidades foi referido que é feita a manutenção preventiva dos equipamentos, com exceção das unidades $F, G$ H, J e L.

O consumo de água das unidades foi analisado através de 17 questões que serão analisadas neste ponto e cujos resultados se encontram resumidos na Tabela 5.

A maioria das torneiras em todas as unidades de alimentação eram de acionamento manual, o que pode promover maior gasto de água (36). Possuíam torneiras com acionamento de pedal para a higienização das mãos numa média de 4 "postos de lavagem de mãos" por unidade. E apenas a unidade B possui torneiras automáticas na área laboral. Verificou-se que a maioria das torneiras das casas de banho eram temporizadas.

No entanto nas unidades $\mathrm{A}, \mathrm{D}$ e H, na casa de banho dos funcionários o acionamento era manual. No caso das unidades B e F não existiam casas de banho para os estudantes, dentro da unidade. Na unidade $\mathrm{C}$ todas as casas de banho tinham torneiras de acionamento manual. A unidade I não possui casas de banho no perímetro.

A substituição das torneiras manuais por automáticas (acionadas por sensor) constituí uma estratégia para evitar gastos por esquecimentos ou torneiras mal calibradas. Isto implica um investimento avultado, mas que teria benefícios tanto económicos como ambientais a longo prazo (36). Verificou-se que a maioria das torneiras e todos os sanitários se encontravam em bom estado de conservação, sem perdas de água, com exceção da unidade J.

Em nenhuma das unidades de alimentação dos SASUP é utilizada água para realizar descongelação, sendo todas as descongelações feitas de um dia para o outro em câmara fria.

Todas as unidades utilizam máquina de lavar loiça, o que é benéfico a nível de redução dos gastos de água, comparativamente à lavagem manual. Tendo em conta serem as mais eficientes, o ideal seria que as unidades maiores ( $A, B, C, D, H$ e L) tivessem máquinas com uma capacidade semelhante à da unidade $\mathrm{C}$ e que as unidades pequenas (E, F, G, I, J) tivessem semelhantes à unidade J. Em todas as unidades as máquinas apenas são usadas quando completamente cheias. O consumo de eletricidade das unidades foi analisado e os resultados encontram-se resumidos na Tabela 5.
Umavezqueamaioria das unidades se localizaemedifícios antigos ou estão integradas nos edifícios das faculdades, não possuem classificação energética. O desperdício médio de energia em Portugal por má eficiência dos edifícios ronda os $136 \mathrm{GWh}$. O conhecimento do estado dos edifícios será o primeiro passo para melhorar estas situações, portanto a obtenção de classificação é já um passo na direção correta. Acresce que o Certificado Energético apresenta medidas de melhoria a serem implementadas que poderiam contribuir para melhorar o desempenho dos edifícios (37).

Em todas as unidades a iluminação é mista (elétrica e natural). A esse nível não se identificou oportunidade de melhoria uma vez que o recurso à iluminação artificial é inevitável. Verificou-se que todas as unidades utilizam lâmpadas fluorescentes que se substituídas por díodos emissores de luz (LED) contribuiriam para reduzir consumos e melhorar a sustentabilidade (38).

A iluminação artificial é controlada maioritariamente de forma manual, exceto na casa de banho do restaurante da unidade de alimentação $H$, que é controlado por sensores de movimento. A opção pela iluminação artificial sempre ligada, do ponto de vista da ambiental, não é claramente a melhor. A melhor opção seria o controlo por sensores de movimento tal como demonstrado por Jennings et al. (39). Na cozinha a melhor opção será manter o controlo manual, tendo em conta as horas de produção e a falta de iluminação natural.

A climatização das unidades está a cargo de unidades de tratamento de ar. Atualmente esta é a opção mais viável uma vez que estas têm a capacidade de aquecer, ventilar e arrefecer o ar, fazendo com que estejam concentrados num só sistema todas as funções de climatização. Estas são ainda capazes de normalizar os níveis de humidade do ar, algo que os sistemas de ar condicionado não conseguem. São também um sistema ecológico, uma vez que a eficiência energética deste tipo de equipamentos tem melhorado e deixaram de utilizar fluidos de refrigeração à base de clorofluorcarbonetos (CFC's), promotores da destruição da camada de ozono, tendo sido substituídos por outros não tóxicos (40, 41).

Não foi possível aferir a classificação energética dos equipamentos elétricos existentes na unidade. A maioria dos equipamentos elétricos são os de cadeia fria e possuem um registador externo de temperatura; os restantes equipamentos possuíam termóstato interno (fornos convetores e fritadeiras).

Do ponto de vista ambiental e da eficiência energética, isto é relevante uma vez que equipamentos que apresentem variações anormais de 


\section{Tabela 5}

Classificações obtidas pelas unidades de alimentação na checklist, nos itens sobre o consumo de água, eletricidade, gás, gestão de resíduos, utilização de hortofrutícolas e avaliação da satisfação dos utentes

\begin{tabular}{|c|c|c|c|}
\hline ITENS & $\begin{array}{l}\text { UNIDADE DE } \\
\text { ALIMENTAÇÃOO }\end{array}$ & $\begin{array}{l}\text { PONTUAÇÃO EM } \\
\text { VALOR ABSOLUTO }\end{array}$ & PONTUAÇÃO (\%) \\
\hline \multirow{11}{*}{ Água } & A & 66 & 65 \\
\hline & B & 47 & 71 \\
\hline & C & 45 & 65 \\
\hline & $\mathrm{D}$ & 57 & 66 \\
\hline & $E$ & 38 & 60 \\
\hline & $\mathrm{F}$ & 26 & 62 \\
\hline & $\mathrm{G}$ & 36 & 67 \\
\hline & $\mathrm{H}$ & 69 & 56 \\
\hline & 1 & 22 & 67 \\
\hline & J & 30 & 71 \\
\hline & L & 42 & 67 \\
\hline \multirow{11}{*}{ Eletricidade } & A & 99 & 73 \\
\hline & $\mathrm{B}$ & 159 & 70 \\
\hline & $\mathrm{C}$ & 280 & 61 \\
\hline & $\mathrm{D}$ & 124 & 84 \\
\hline & E & 105 & 71 \\
\hline & $\mathrm{F}$ & 32 & 53 \\
\hline & $G$ & 107 & 75 \\
\hline & $\mathrm{H}$ & 231 & 50 \\
\hline & 1 & 24 & 62 \\
\hline & J & 93 & 76 \\
\hline & L & 137 & 80 \\
\hline \multirow{11}{*}{ Gás } & A & 59 & 56 \\
\hline & $\mathrm{B}$ & 57 & 54 \\
\hline & $\mathrm{C}$ & 53 & 61 \\
\hline & $\mathrm{D}$ & 57 & 54 \\
\hline & E & 41 & 55 \\
\hline & $\mathrm{F}$ & 15 & 50 \\
\hline & $\mathrm{G}$ & N/A & N/A \\
\hline & $\mathrm{H}$ & 26 & 51 \\
\hline & 1 & $\mathrm{~N} / \mathrm{A}$ & $\mathrm{N} / \mathrm{A}$ \\
\hline & J & 16 & 59 \\
\hline & $L$ & 71 & 61 \\
\hline \multirow{11}{*}{$\begin{array}{l}\text { Gestão de } \\
\text { resíduos }\end{array}$} & A & 54 & 78 \\
\hline & $\mathrm{B}$ & 50 & 72 \\
\hline & $\mathrm{C}$ & 50 & 72 \\
\hline & $\mathrm{D}$ & 50 & 72 \\
\hline & $\mathrm{E}$ & 50 & 72 \\
\hline & $\mathrm{F}$ & 19 & 63 \\
\hline & $\mathrm{G}$ & 32 & 71 \\
\hline & $\mathrm{H}$ & 51 & 74 \\
\hline & 1 & 49 & 71 \\
\hline & $\mathrm{J}$ & 54 & 78 \\
\hline & L & 32 & 71 \\
\hline \multirow{11}{*}{$\begin{array}{l}\text { Utilização de } \\
\text { hortofrutícolas }\end{array}$} & A & 18 & 60 \\
\hline & B & 18 & 60 \\
\hline & C & 18 & 60 \\
\hline & $D$ & 18 & 60 \\
\hline & $\mathrm{E}$ & 18 & 60 \\
\hline & $\mathrm{F}$ & 18 & 60 \\
\hline & $\mathrm{G}$ & 12 & 50 \\
\hline & $\mathrm{H}$ & 18 & 60 \\
\hline & 1 & 12 & 50 \\
\hline & $\mathrm{J}$ & 12 & 40 \\
\hline & L & 18 & 60 \\
\hline \multirow{11}{*}{$\begin{array}{l}\text { Avaliação da } \\
\text { satisfação dos } \\
\text { Utentes }\end{array}$} & A & 41 & 68 \\
\hline & B & 62 & 67 \\
\hline & C & 62 & 67 \\
\hline & D & 62 & 67 \\
\hline & $\mathrm{E}$ & 61 & 68 \\
\hline & $\mathrm{F}$ & 41 & 68 \\
\hline & $\mathrm{G}$ & 35 & 83 \\
\hline & $\mathrm{H}$ & 41 & 68 \\
\hline & 1 & 35 & 83 \\
\hline & $\mathrm{J}$ & 35 & 83 \\
\hline & L & 57 & 68 \\
\hline
\end{tabular}

temperatura encontram-se com algum tipo de avaria quer seja na geração de energia quer no sistema de vedação, o que irá levar a um gasto extra de energia (42).

As unidades que se encontram classificadas como "Não Aplicável" (N/A) são as que não possuem ligação de gás nas cozinhas (Tabela 5). Existem unidades sem ligação de gás, uma vez que recebem as refeições prontas a servir (unidades $\mathrm{G}$ e I). A unidade A partilha o contador do gás com a sede dos SASUP.

Em relação ao tipo de gás utilizado, a maioria das unidades utiliza gás natural. Apenas as unidades $\mathrm{B}, \mathrm{D}$ e $\mathrm{F}$ utilizam gás propano. $\mathrm{Na}$ perspetiva ambiental, o gás natural é a melhor opção, uma vez que apesar de ter menor capacidade calorifica que o propano e o butano, a sua combustão liberta menos dióxido de carbono. Isto torna-o a opção mais "limpa" das 3, sendo que a sua utilização significa uma pegada de carbono menor. Tem ainda uma densidade mais baixa que o ar, facilitando a sua dissipação e um nível de humidade quase nulo, que dificulta a sua combustão, tornando-o mais seguro em caso de fuga (43). As unidades de alimentação que utilizam gás propano deviam proceder à substituição por gás natural que é mais económico e melhor a nível ambiental.

Não foi possível aferir a classificação energética dos equipamentos a gás existentes na unidade. Os únicos equipamentos possuíam um termóstato interno e os fornos de convecção mais recentes tinham ambos, termóstato interno e externo.

A gestão de resíduos foi analisada e os resultados encontram se resumidos na Tabela 5 .

Em todas as unidades são utilizados equipamentos de proteção individual no manuseamento de detergentes e desinfetantes. Todos os produtos estão identificados e possuem ficha técnica e de dados de segurança, com exceção da unidade $F$.

Os produtos usados eram maioritariamente não-biodegradáveis (6 produtos no geral), 2 eram corrosivos/inflamáveis e 1 biodegradável nas unidades que estavam concessionadas e 3 corrosivos/inflamáveis nas unidades sob gestão dos SASUP.

A dosagem dos produtos de higienização é feita na maioria das unidades através de doseador manual, exceto nas unidades A, G, J e L. A dosagem automática é vantajosa uma vez que diminui gastos desnecessários e a quantidade descartada na rede de saneamento básico (44). A dosagem do sabão para higienização das mãos era feita por dosagem manual em todas as unidades.

Todas as unidades, com exceção da unidade J, fazem a separação de resíduos e realizam a reciclagem de óleos, paletes e outros.

Nenhuma das unidades utiliza material reciclado nas suas operações, algo que poderia ser repensado no que toca principalmente ao papel e plásticos

Apenas são enviadas para o lixo orgânico as sobras da preparação e do prato, exceto a unidade J. Em nenhuma das unidades é registada a quantidade de sobras.

Todas as unidades usam produtos/materiais descartáveis, sendo que os mais comuns são luvas, kits para visitantes e guardanapos. Nas unidades A, E, H, I, J e L também são usados aventais e toucas descartáveis e nas F, l e G são usadas toalhas de papel, sendo ainda que na unidade G também são utilizados talheres e pratos descartáveis. Os guardanapos, kits para visitantes e luvas são indispensáveis. Os restantes materiais como as toalhas de papel, aventais, toucas, talheres e os pratos descartáveis podem ser substituídos por não descartáveis (45).

Nenhuma das unidades possui trituradora de resíduos orgânicos ou inorgânicos. A instalação de pelo menos uma trituradora de resíduos orgânicos por unidade iria ajudar a reduzir o lixo produzido. As sobras podem ser encaminhadas para a produção de adubo orgânico, para as 
áreas verdes das faculdades, como é exemplo desde 1995 a University of Northern British Columbia, no Canadá (46, 47).

Quase todas as unidades possuem caixa de separação de gordura na canalização, com exceção das unidades G, I e J que permite separar a gordura da água, reduzindo os entupimentos por acumulação de gordura nas tubagens (48).

A utilização de hortofrutícolas foi analisada e os resultados encontram se resumidos na Tabela 5.

Os fornecedores dos SASUP fornecem os hortícolas maioritariamente in natura, o que é positivo a nível da produção de resíduos inorgânicos, uma vez que apesar de a produção de cascas, caroços e outros resíduos aumentar, são utilizadas menos embalagens de plástico. Os produtos adquiridos não são biológicos nem exclusivamente provenientes de produtores locais ou nacionais.

As quantidades gastas pelos SASUP poderiam ser garantidas por fornecedores nacionais e contribuir para a economia local e redução da pegada de $\mathrm{CO}_{2}$, tal como tem sido feito na Ball State University, nos EUA (49).

Tendo em conta o peso da agricultura não biológica para o meio ambiente, referidas por Pimentel et al. (50) à medida que as condições para a obtenção deste tipo de produtos for melhorando sugere-se a sua inclusão progressiva nas refeições.

A Tabela 5 apresenta os resultados referentes às classificações obtidas pelas unidades de alimentação na checklist nos itens sobre avaliação de satisfação dos utentes.

A avaliação da satisfação do utente é responsabilidade dos SASUP em todas as unidades de alimentação e é feita por meio da aplicação de inquéritos de satisfação. Existem ainda livros de reclamações, que de forma indireta permitem avaliar a satisfação com o serviço pelo número, tipo e frequência das queixas.

Na maioria das unidades de alimentação é possível fazer a compra por item de refeição, ou seja, comprar ou só a sopa ou só o prato com exceção das unidades B, D, E e I. A falta desta opção pode levar à perda de utentes ou contribuir para o desperdício alimentar, tal como referido por Ferreira et al. (51).

Um aspeto positivo, em todas as unidades é a possibilidade de pedir ajustes na quantidade de comida no empratamento, conforme o desejo do utente o que contribui para a satisfação do cliente.

Algumas unidades de alimentação utilizam a marcação prévia de refeição ( $F, G$, I e J) com diferentes tipos de penalização. A marcação prévia de refeições, pode ser uma boa ferramenta de gestão para evitar a produção desnecessária de refeições, podendo assim contribuir para reduzir o desperdício e aumentar a satisfação do utente, tal como é constatado por Ferreira et al. (51).

\section{CONCLUSÕES}

Os consumos de água, eletricidade e gás não são registados pelo que não estão estabelecidas metas de forma a potenciar a redução do desperdício ou controlo dos gastos. A maioria das unidades localiza-se em edifícios antigos não possuindo classificação energética. Esta situação aponta para a necessidade de intervenção e investimento no sentido de controlo e redução de gastos.

Todos os produtos de higienização eram maioritariamente não-biodegradáveis mas possuíam ficha técnica e dados de segurança.

A maioria das unidades faz a separação de resíduos e a reciclagem de óleos, paletes e outros. Nenhuma possui trituradora de resíduos orgânicos ou inorgânicos e todas utilizam produtos/materiais descartáveis, Em nenhuma das unidades é registada a quantidade de sobras.

Na maioria das unidades é feita a manutenção preventiva dos equipamentos.
Através da análise dos resultados obtidos na checklist, concluiu-se que todas se encontravam acima do limite de Aceitável. As unidades dos SASUP para melhorar o seu estado geral de sustentabilidade terão de efetuar investimento económico em equipamentos e infraestruturas e simultaneamente sensibilizar/ formar os trabalhadores e gestores no sentido de mudança de atitude/comportamento em prol de reduzir o impacto ambiental das unidades.

A ferramenta desenvolvida pode constituir um apoio à gestão ambiental e à melhoria das condições de sustentabilidade das unidades de alimentação coletiva.

\section{AGRADECIMENTOS}

This research was supported by national funds through FCT - Foundation for Science and Technology within the scope of UIDB/05748/2020 and UIDP/05748/2020.

\section{REFERÊNCIAS BIBLIOGRÁFICAS}

1. Strasburg VJ, Jahno VD. Paradigmas das práticas de gestão ambiental no segmento de produção de refeições no Brasil: Práticas de gestão ambiental na produção de refeições. Revista Engenharia Sanitária e Ambiental. 2015.

2. Abreu ESd, Spineli MGN, Pinto AMdS. Gestão de Unidades de Alimentação e Nutrição: Um Modo de Fazer. $4^{a}$ ed. São Paulo: Metha; 2011. 351 p.

3. Veiros MB, Proença RPC. Princípios de Sustentabilidade na Produção de Refeições. Nutrição em Pauta. 2010;102:45-9.

4. Wang R. Investigations of Important and Effective Effects of Green Practices in Restaurants. Procedia - Social and Behavioral Sciences. 2012;40:94-8.

5. Pirani SI, Arafat HA. Solid waste management in the hospitality industry: A review. Journal of Environmental Management. 2014(146):320-36.

6. Nations FaAOotU. Energy-smart food for people and climate. Italy: United Nations; 2011. 7. Harmon A, Gerald B, Association AD. Position of the American Dietetic Association: Food and Nutrition Professionals Can Implement Practices to Conserve Natural Resources and Support Ecological Sustainability. Journal of the American Dietetic Association. 2007;107(6):1033-43.

8. Tagtow A, Robien K, Bergquist E, Bruening M, Dierks L, Hartman BE, et al. Academy of Nutrition and Dietetics: Standards of Professional Performance for Registered Dietitian Nutritionists (Competent, Proficient, and Expert) in Sustainable, Resilient, and Healthy Food and Water Systems. Journal of the Academy of Nutrition and Dietetics. 2014;114(3):475 - 88.

9. (FECOMERCIO) FdCdEdSP. O uso racional da água no comércio2010:[56 p.]. Available from: http://site.sabesp.com.br/uploads/file/asabesp_doctos/cartilha_fecomercio.pdf. 10. Souza DPd, Santos RK, Santos RF. Estimativa do consumo de água em restaurantes na cidade de Cascavel - PR. Acta Iguazu. 2012;1(3):21-30.

11. Barthichoto M, Matias ACG, Spinelli MGN, Abreu ESd. Responsabilidade Ambiental: Perfil das Práticas de Sustentabilidade Desenvolvidas em Unidades Produtoras de Refeições do Bairro de Higienópolis, Município de São Paulo. Qualit@s Revista Eletrônica. 2013;14(1):1-12.

12. Lang T, Barling D, Caraher M. Food Policy: Integrating Health, Environment and Society: Oxford University Press; 2009 12/11. 336 p.

13. Portugal Ed. Fator de Emissão Anual https://energia.edp.pt/particulares/apoiocliente/simulador-co2/2016.

14. Madeira EdEd. Método de Cálculo de Emissão de CO2 https://www.eem.pt/pt/ conteudo/sustentabilidade/rotulagem-de-energia-el\%C3\%A9ctrica/m\%C3\%A9todode-c\%C3\%A1lculo-das-emiss\%C3\%B5es-de-co2-e-de-outros-gases-com-impactesambientais/2017.

15. Yu Y, Zhao J, Bayly AE. Development of Surfactants and Builders in Detergent Formulations. Chinese Journal of Chemical Engineering. 2008;16(4):517-27.

16. Scott MJ, Jones MN. The biodegradation of surfactants in the environment. Biochimica et Biophysica Acta (BBA) - Biomembranes. 2000;1508(1-2):235-51.

17. Authority EP. The Disposal of Soaps and Detergents. Austrália: Environment Protection Authority; 2004. p. 3. 
18. Zeng SX, Meng XH, Yin HT, Tam CM, Sun L. Impact of cleaner production on business performance. Journal of Cleaner Production. 2010;18(10-11):975-83.

19. Alonso-Almeida MdM, Rodríguez-Antón JM, Rubio-Andrada L. Reasons for implementing certified quality systems and impact on performance: an analysis of the hotel industry. The Service Industries Journal. 2012;32(6):919-36.

20. Association GR. Green Restaurant Certification 4.0 Standards Boston: Green Restaurante Association; 2008 [Available from: www.dinegreen.com/certificationstandards.

21. Moretti GN, Sautte KD, Azevedo JAM. ISO 14001: implementar ou não? Uma proposta para a tomada de decisão. Revista Engenharia Sanitária e Ambiental. 2008 13(4):416-25.

22. Neto AS, Campos LM, Shigunov T. Fundamentos da Gestão Ambiental. Rio de Janeiro: Ciência Moderna; 2009. 295 p.

23. Shrivastava P. The Role of Corporations in Achieving Ecological Sustainability. The Academy of Management Review. 1995.

24. Heller M, Keoleian G. Life Cycle-Based Sustainability Indicators For Assessment of the U.S. Food System. Michigan: The Center for Sustainable Systems; 2000.

25. Souza D. Comunicação Organizacional e Responsabilidade Social Corporativa: A Construção dos Conceitos. Minas Gerais: Universidade Federal de Minas Gerais; 2003. 26. Porto CEdSdASdUd. Regulamento Orgânico dos Serviços de Acção Social da Universidade do Porto2015:[15 p.]. Available from: https://sigarra.up.pt/sasup/pt/ web_base.gera_pagina?P_pagina=267389

27. Porto SdASdUd. Relatório de Atividades e Contas2015. Available from: https://sigarra.up.pt/sasup/pt/SAS_UTIL.VER_DOCS?pct_pag_id=251872\&pct_ parametros=?\&pct_grupo=467\&pct_grupo=997\&pct_grupo=789.

28. EXAME. Guia EXAME de Sustentabilidade 2016 - Questionário Dimensão Ambiental. 2016

29. Europeia C. Checklist for inspection of waste management operation (LT). Pratical Manual2005.

30. Green Seal I. Green Seal Standard for Restaurants and Food Services2014. Available from: http://www.greenseal.org/Portals/O/Documents/Standards/GS-55/ GS-55_Ed1-0_Restaurants_and_Food_Services.pdf.

31. Institution BS. Self-assessment questionnaire: How ready are you for ISO 14001 2004.

32. University CfSTaEC. Sustainable Tourism Practices Checklist. 2007.

33. Zak J. Sustainable Operation of Shopping Centers2010. Available from: http://www. ece.com/fileadmin/PDF_englisch/Unternehmensbroschueren/Manual_Sustainable_ Operation_of_Shopping_Centers.pdf.

34. Zambrano TF, Martins MF. Utilização do método FMEA para avaliação do risco ambiental. Gestão e Produção. 2007;14(2):295 - 309.

35. Mikkola M. Shaping professional identity for sustainability. Evidence in Finnish public catering. Appetite. 2009;53(1):56-65.

36. Harmon A. Do automatic water faucets actually save water? A comparative test of manual and automatic water faucets. Sacramento: California State University; 2016. 37. Silva VPPd. Reabilitação Energética de Edifícios Residenciais: Propostas de intervenção numa perspetiva de otimização da relação custo/benefício: Universidade de Aveiro; 2013.

38. Khorasanizadeh H, Parkkinen J, Parthiban R, Moore JD. Energy and economic benefits of LED adoption in Malaysia. Renewable and Sustainable Energy Reviews. 2015;49:629-37.

39. Jennings JD, Rubinstein FM, DiBartolomeo D, Blanc SL. Comparison of Control Options in Private Offices in an Advanced Lighting Controls Testbed. Journal of the Illuminating Engineering Society. 2000:27.

40. Brito VEFd. Tratamento e distribuição do ar em sistemas de climatização: Universidade de Coimbra; 2010.

41. Matos RS. Manual Didático de Refrigeração. 2004.

42. Jorge JGRAB. O Impacto De Boas Práticas De Gestão E Utilização Dos Sistemas De Refrigeração Na Eficiência Energética: Universidade Do Porto; 2015.

43. Mesquita L. Rede de Gás: Instituto Politécnico de Bragança; 2007.

44. Farias RM. Manual De Segurança Na Higiene E Limpeza. $1^{a}$ edição ed2011. 480 p.
45. Gallardo A, Edo-Alcón N, Carlos M, Renau M. The determination of waste generation and composition as an essential tool to improve the waste management plan of a university. Waste Management. 2016;53:3-11.

46. Smyth DP, Fredeen AL, Booth AL. Reducing solid waste in higher education: The first step towards 'greening' a university campus. Resources, Conservation and Recycling. 2010;54(11):1007-16.

47. Pardo M, Santiago A, Pozo A, Estuardo D. Estudio del proceso de trituración de los residuos sólidos orgánicos para reducir la contaminación residual en el mercado mayorista de Ambato: Universidad Técnica de Ambato; 2013.

48. Faria J, Sousa A, Reis A, Filipe V, Barroso J. Probe and Sensors Development for Level Measurement of Fats, Oils and Grease in Grease Boxes. Sensors. 2016;16(1517):0 - 20 .

49. Koester RJ, Eflin J, Vann J. Greening of the campus: a whole-systems approach. Journal of Cleaner Production. 2006;14(9):769-79.

50. Pimentel D, Hepperly P, Hanson J, Douds D, Seidel R. Environmental, Energetic, and Economic Comparisons of Organic and Conventional Farming Systems. BioScience. 2005;55(7):573-82.

51. Ferreira M, Martins ML, Rocha A. Food waste as an index of foodservice quality. British Food Journal. 2013;115(11):1628-37. 